\title{
Atenção farmacêutica no uso de métodos contraceptivos: uma revisão narrativa
}

\author{
Pharmaceutical care in the use of contraceptive methods: a narrative review \\ Atención farmacéutica en el uso de métodos anticonceptivos: una revisión narrativa
}

Recebido: 16/11/2021 | Revisado: 23/11/2021 | Aceito: 26/11/2021 | Publicado: 09/12/2021

\author{
Andreza Kalline Rocha da Silva \\ ORCID: https://orcid.org/0000-0001-7041-0900 \\ Faculdade de Palmas, Brasil \\ E-mail: andrezakalliny2016@gmail.com \\ Rafaela Rocha Pinto \\ ORCID: https://orcid.org/0000-0001-6922-7332 \\ Faculdade de Palmas, Brasil \\ E-mail: rafaela.pinto5@docente.suafaculdade.com.br
}

\begin{abstract}
Resumo
Objetivo: Discutir a atenção farmacêutica quanto ao uso de métodos contraceptivos. Método: Foi realizada uma revisão narrativa da literatura com consulta as publicações entre os anos de 2000 a 2021 em plataformas virtuais. Resultados: Todos os produtos e métodos estão associados a benefícios e complicações potenciais que devem ser considerados com o paciente e os profissionais de saúde na seleção da opção mais satisfatória. A atenção farmacêutica nas orientações aos métodos contraceptivos deve começar averiguando o histórico de saúde da paciente, identificando condições ou características relacionadas à segurança do contraceptivo hormonal. Assim, os farmacêuticos fornecem aconselhamento medicamentoso e educação em saúde à medida que o paciente inicia sua avaliação contraceptiva e contínua adesão e tolerabilidade durante as entregas subsequentes. Conclusão: Existe uma ampla variedade de medicamentos empregados na contracepção, e isso pode provocar nos pacientes muitas dúvidas em relação às melhores escolhas ao uso. Por isso o relacionamento entre farmacêutico e paciente torna-se imprescindível para uma farmacoterapia bem sucedida. É fundamental que os farmacêuticos passem informações claras sobre a melhor forma de realizar o tratamento com métodos contraceptivos, além disso, esclareça sobre as formas corretas do uso de medicamentos mostrando também as possíveis contraindicações e interações medicamentosas, contribuindo de forma efetiva, para minimizar os riscos da medicação e reações adversas. A atenção farmacêutica promoverá, portanto, um atendimento personalizado, humanizado e cientificamente correto sobre o uso dos métodos contraceptivos.
\end{abstract}

Palavras-chave: Assistência farmacêutica; Boas práticas farmacêuticas; Fármacos anticoncepcionais.

\begin{abstract}
Objective: Discuss pharmaceutical care regarding the use of contraceptive methods. Method: A narrative literature review was carried out, consulting publications between 2000 and 2021 on virtual platforms. Results: All products and methods are associated with potential benefits and complications that must be considered with the patient and healthcare professionals in selecting the most satisfactory option. Pharmaceutical care in guidelines for contraceptive methods should begin by investigating the patient's health history, identifying conditions or characteristics related to the safety of the hormonal contraceptive. Thus, pharmacists provide drug counseling and health education as the patient begins their contraceptive evaluation and continued adherence and tolerability during subsequent deliveries. Conclusion: There is a wide variety of medications used in contraception, and this can cause patients to have many doubts about the best choices for use. Therefore, the relationship between pharmacist and patient becomes essential for successful pharmacotherapy. It is essential that pharmacists provide clear information about the best way to carry out the treatment with contraceptive methods, in addition to clarifying the correct ways to use drugs, also showing possible contraindications and drug interactions, effectively contributing to minimize the risks medication and adverse reactions. Therefore, pharmaceutical care will promote personalized, humanized and scientifically correct care regarding the use of contraceptive methods.
\end{abstract}

Keywords: Pharmaceutical assistance; Good pharmaceutical practices; Contraceptive drugs.

\section{Resumen}

Objetivo: Discutir la atención farmacéutica con respecto al uso de métodos anticonceptivos. Método: Se realizó una revisión de literatura narrativa, consultando publicaciones entre 2000 y 2021 en plataformas virtuales. Resultados: Todos los productos y métodos están asociados con potenciales beneficios y complicaciones que deben ser considerados con el paciente y los profesionales de la salud en la selección de la opción más satisfactoria. La atención farmacéutica en las pautas para los métodos anticonceptivos debe comenzar investigando el historial de salud de la paciente, identificando condiciones o características relacionadas con la seguridad del anticonceptivo hormonal. Por lo tanto, los farmacéuticos brindan asesoramiento sobre medicamentos y educación para la salud a medida que la paciente comienza 
su evaluación anticonceptiva y la adherencia y tolerabilidad continuas durante los siguientes partos. Conclusión: Existe una amplia variedad de medicamentos utilizados en la anticoncepción, y esto puede hacer que los pacientes tengan muchas dudas sobre las mejores opciones de uso. Por lo tanto, la relación entre el farmacéutico y el paciente se vuelve esencial para una farmacoterapia exitosa. Es fundamental que los farmacéuticos brinden información clara sobre la mejor forma de realizar el tratamiento con métodos anticonceptivos, además de aclarar las formas correctas de uso de los medicamentos, mostrando también las posibles contraindicaciones e interacciones medicamentosas, contribuyendo de manera efectiva a minimizar los riesgos de medicación y reacciones adversas. Por tanto, la atención farmacéutica promoverá una atención personalizada, humanizada y científicamente correcta en cuanto al uso de métodos anticonceptivos.

Palabras clave: Asistencia farmacéutica; Buenas prácticas farmacéuticas; Anticonceptivos.

\section{Introdução}

As estimativas mostram que população mundial total esperada pode atingir 9,51 bilhões até o ano de 2050; com isso, a saúde sexual e reprodutiva têm afetado muito as mulheres e adolescentes, especialmente nos países em desenvolvimento. Todos os anos, mais de 120 milhões de casais têm uma necessidade não atendida de contracepção, 80 milhões de mulheres têm gravidez não desejada, sendo que 45 milhões dessas mulheres terminam em aborto. Nesse cenário, mais de meio milhão de mulheres morrem por complicações associadas à gravidez, parto e ao período pós-parto e 340 milhões de pessoas adquirem novas infecções sexualmente transmissíveis, entre outras questões urgentes que certamente precisam de maior atenção dos profissionais de saúde, incluindo farmacêuticos (Chin, 2011).

Convém destacar que a contracepção é definida como a prevenção intencional da concepção através do uso de diversos dispositivos, práticas sexuais, produtos químicos, drogas ou procedimentos cirúrgicos. Assim, qualquer dispositivo ou ato cujo propósito é evitar que o casal procrie pode ser considerado como um método contraceptivo (Jain \& Muralidhar, 2011; Brasil, 2013).

Em qualquer contexto social, a contracepção eficaz permite que um casal desfrute de uma relação física sem medo de uma gravidez indesejada e garante liberdade suficiente para ter filhos quando desejado. Um desafio para a saúde pública é ofertar métodos contraceptivos com o máximo de conforto e privacidade, e ao mesmo tempo com custos mínimos, poucos efeitos colaterais e alta adesão. Alguns métodos de barreira, como preservativos masculinos e femininos, também proporcionam dupla vantagem da proteção contra infecções sexualmente transmissíveis (Brasil, 2013).

O uso da contracepção é um avanço no direito humano. Discutir sobre a importância do uso dos métodos contraceptivos e suas vantagens e desvantagens são fundamentais para o planejamento familiar. O Brasil é um país que enfrenta um grande problema que é a gravidez indesejada. A gravidez indesejada, na maioria das vezes, é finalizada com a prática de aborto não seguro, que poderia ser evitado caso o casal houvesse feito uso de algum método contraceptivo (Vieira, 2010).

O planejamento familiar conforme a Lei Federal 9.263/96 é direito de todo o cidadão e se caracteriza pelo conjunto de ações de regulação da fecundidade que garanta direitos iguais de constituição, limitação ou aumento da prole pela mulher, pelo homem ou pelo casal (Brasil, 1996). O planejamento familiar consiste em dar à família o direito de ter quantos filhos quiser, no momento que lhe for mais conveniente, com toda a assistência necessária para garantir integralmente o exercício do direito ao planejamento familiar (Dantas, et al., 2014).

De acordo com a Organização Mundial de Saúde, entre os 1,9 bilhões de mulheres da faixa etária reprodutiva (15 a 49 anos) em todo o mundo em 2019, 1,1 bilhão almejam o planejamento familiar, sendo que destas, 842 milhões usam métodos contraceptivos e 270 milhões têm uma necessidade não atendida de contracepção (Who, 2019).

Atualmente o Sistema Único de Saúde (SUS) oferta métodos contraceptivos a população, com fácil acesso e informações ao casal para auxiliá-los na escolha daquele que melhor se adapta a sua realidade, destacando também a importância do conhecimento dos profissionais de saúde, sobre as orientações e informações pertinentes a esse tema (Leal \& Rodrigues, 2019). 
Nessa direção, a atenção farmacêutica tem se expandido, e o profissional farmacêutico é considerado fundamental para prestar orientações, palestras, sanar dúvidas entre outras atividades inerentes à utilização dos métodos contraceptivos. Leal e Rodrigues (2019) ressaltam que a atenção farmacêutica foi determinada pelo Conselho Nacional de Saúde na resolução 338/2004 como um conjunto de ações voltadas à promoção, proteção e recuperação da saúde, tanto individual como coletivo, tendo o medicamento como insumo essencial e visando o acesso e seu uso racional.

Os farmacêuticos têm demonstrado maior atenção e interesse em cuidados de saúde sexual e reprodutiva, domínio que ainda carece de participação ativa da profissão de farmácia. Reconhecer e atender às necessidades das pessoas em saúde sexual e reprodutiva são tão importantes quanto fornecer assistência farmacêutica em outras disciplinas. A saúde sexual e reprodutiva não deve ser negligenciada pela profissão da farmácia, pois se expande de seu papel tradicional de dispensação para serviços mais centrados no paciente. Investigações de práticas de dispensação e aconselhamento de farmacêuticos sobre o fornecimento de contraceptivos também estão sendo estudadas ativamente. A capacidade dos farmacêuticos de dispensar e educar o público, especialmente adolescentes, sobre a prevenção de gravidez indesejada e infecção sexualmente transmissível através do uso de métodos contraceptivos tem sido amplamente estudada (Chin, 2011; Leal \& Rodrigues, 2019).

Leal e Rodrigues (2019) destacam que entre as diversas atribuições do farmacêutico, a orientação em relação à farmacoterapia favorece a qualidade de vida e eficácia na terapia medicamentosa. Isso tudo faz com que o farmacêutico seja o profissional de saúde mais indicado e preparado para a orientação quanto ao uso de medicamentos contraceptivos.

Diante do exposto, este trabalho tem como objetivo discutir a atenção farmacêutica quanto ao uso de métodos contraceptivos, descrevendo os tipos mais usados na atualidade, suas formas de uso, indicações, contraindicações e possibilidades de reversibilidade.

\section{Metodologia}

Esse estudo foi realizado através de uma revisão narrativa da literatura onde a atenção farmacêutica foi discutida em relação aos métodos contraceptivos mais utilizados e recomendados. Nessa direção, de forma simples e pontual, foi realizada uma síntese dos conhecimentos e experiências de estudos empíricos através dos meios eletrônicos, livros-textos, artigos acadêmicos, buscando assim, trabalhos publicados sobre a temática.

A revisão narrativa possibilita sintetizar o conhecimento sobre um determinado assunto, promovendo o aprofundamento dos conhecimentos e levando ao pensamento crítico, pontos relevantes à prática diária em saúde (Barros \& Lehfeld, 2000).

A pergunta norteadora da pesquisa foi: "O que mostram os artigos científicos sobre a atenção farmacêutica quanto ao uso de contraceptivos?". Os trabalhos revisados no presente estudo foram identificados por meio das seguintes bases de dados: Plataforma da Biblioteca Virtual em Saúde (BVS), Scientific Electronic Library Online (SciELO), Google Acadêmico e demais literatura técnicas do Ministério da Saúde (cartilhas e informações e/ou documentos).

Foram incluídas publicações entre os anos de 2000 a 2021. As palavras-chave buscadas no idioma português com uso do operador booleano AND foram: "assistência farmacêutica", "boas práticas farmacêuticas", "padrões de prática do profissional farmacêutico", "agentes contraconceptivos", "fármacos anticoncepcionais", "planejamento familiar", "relações profissionalpaciente". Os resultados e discussão estão apresentados através de uma narração descritiva. (fonte: TNR 10 - justificado - espaço $1,5)$.

\section{Resultados e Discussão}

\subsection{Os métodos contraceptivos}

A história da contracepção é milenar. Há registros de métodos contraceptivos dos antigos egípcios, há mais de mil anos antes de Cristo. Nesse sentido, a prevenção da gravidez continua sendo uma parte importante da prática dos cuidados em saúde. 
A contracepção pode ocorrer em vários pontos do processo biológico reprodutivo e através de uma série de opções de produtos contraceptivos. Os farmacêuticos são prestadores de cuidados de saúde devidamente habilitados para auxiliar os pacientes na seleção adequada de produtos contraceptivos com base em suas situações pessoais e estilos de vida (Colquitt \& Martin, 2017).

Os produtos contraceptivos são categorizados pelo seu conteúdo hormonal e método de ação. As opções hormonais incluem pílulas anticoncepcionais orais, adesivos anticoncepcionais, implantes, injeção, dispositivos intravaginais e intrauterinos. Os produtos de barreira previnem a gravidez criando um obstáculo físico para a fertilização bem sucedida de um óvulo pelos espermatozoides. Todos os produtos e métodos estão associados a benefícios e complicações potenciais que devem ser considerados com o paciente e os profissionais de saúde na seleção da opção mais satisfatória (Colquitt \& Martin, 2017).

No entanto, existem efeitos colaterais muito comuns associados ao uso de contraceptivos, entre estes se destacam: tonturas, náuseas, dores de cabeça, irritabilidade, miastalgia, vômitos, aumento do apetite com consequente ganho de peso, queda de cabelo e alterações no apetite sexual (Almeida \& Assis, 2017).

$\mathrm{O}$ uso de alguns contraceptivos, entre eles os por via oral, também aumenta o risco de doença cardiovascular, em particular o risco de tromboembolismo venoso, enfarte do miocárdio e acidente vascular cerebral. O risco de doença grave ou morte atribuível ao uso de contraceptivos orais por efeitos cardiovasculares adversos concentra-se principalmente entre mulheres com mais de 30 anos e que apresente algum fator de risco cardiovascular, como por exemplo, ser ou já ter sido fumante (Novosartyan, 2021).

Existem muitos métodos contraceptivos disponíveis no Brasil, incluindo implantes, dispositivos intrauterinos (DIU) de cobre ou hormonal, injeções, pílulas, anéis vaginais, métodos de barreira (preservativos masculinos e femininos e diafragmas), esterilização feminina e masculina, contracepção de emergência e métodos naturais (planejamento familiar natural) (Brasil, 2013).

A contracepção reversível de longa duração promove a contracepção segura e eficaz ao longo de vários anos. Os métodos reversíveis são os mais eficazes disponíveis atualmente e requerem substituição com menos frequência quando comparados aos outros métodos. Os principais métodos reversíveis são o implante contraceptivo e o DIU de cobre ou hormonal (Brandão, 2019).

O implante contraceptivo é uma pequena haste de plástico que é colocada sob a pele do braço. Esse implante libera lentamente uma dose baixa do hormônio progesterona, que impede os ovários de liberar um óvulo a cada mês. Geralmente ocorre uma mudança no período menstrual da mulher e, em alguns casos, ela pode ficar sem menstruar. O implante é considerado bastante seguro e dura em média três anos, podendo ser facilmente ser removido (Braga \& Vieira, 2015).

O dispositivo intrauterino contraceptivo (DIU) é um pequeno dispositivo implantado no útero. Existem dois tipos de DIU, o de cobre que dura de 5 a 10 anos, e o de liberação hormonal que dura 5 anos em média. O DIU impede que o espermatozoide alcance e fertilize um óvulo. Ambos os DIUs são seguros e podem ser utilizados durante a amamentação, além disso, não fazem interações medicamentosas com outros medicamentos (Who, 2019).

Os métodos contraceptivos reversíveis de longa duração são superiores em termos de eficácia, propiciando taxas de gravidez de menos de $1 \%$ ao ano em uso perfeito e em uso típico. Uma das principais vantagens em relação aos contraceptivos reversíveis de curta duração é a manutenção da sua alta eficácia, independentemente da motivação da usuária. Esses métodos independem da ação do médico ou da usuária para manter sua eficácia e apresentam as mais altas taxas de satisfação e continuidade de uso entre todos os contraceptivos reversíveis. O implante contraceptivo de etonogestrel, único disponível no Brasil, apresenta taxa de falha de $0,05 \%$, com duração de 3 anos. Para efeito de comparação, o dispositivo intrauterino de cobre (DIU), que também é bastante eficaz como contraceptivo, tem taxa de falha variando entre $0,6 \%$ e $0,8 \%$ no primeiro ano de uso (Junges, et al., 2021).

A injeção contraceptiva (Depo-Provera ${ }^{\mathrm{TM}}$ ou Depo-Ralovera ${ }^{\mathrm{TM}}$ ) é a base de progestina sintética (hormônio progesterona 
sintético) que inibe a secreção das gonadotrofinas (hormônio responsável pelo ciclo menstrual), evitando a ovulação (processo em que o óvulo segue do ovário para o útero, onde pode ser fecundado), e reduzindo a espessura do endométrio (camada que recobre internamente o útero). É um método contraceptivo muito eficaz, com apenas $0,1 \%$ a $0,6 \%$ de falha para a injeção mensal e de $0,3 \%$, para a injeção trimestral, o que é equivalente à eficácia da ligadura de trompas. Apesar de possui uma alta eficácia existe algumas desvantagens tais como: requer a aplicação por um profissional da saúde; é irreversível; exige controle do número de semanas que foi usado; não protege contra infeções sexualmente transmissíveis; aumenta o risco de cefaleia, alterações no humor aumentam o peso, dor abdominal, ciclos menstruais irregulares. Pode ser usado durante a amamentação e não é afetado por outros medicamentos (Farias, et al., 2006; Lopes, et al., 2020; Junges, et al., 2021).

A contracepção hormonal combinada contém formas sintéticas dos hormônios estrogênio e progesterona. Está disponível com uma prescrição como pílula (contracepção oral) ou anel vaginal. Esses métodos são muito eficazes (99,5\%) se usados da maneira certa. Geralmente não são recomendados para aqueles que estão em risco de doenças cardíacas ou fumantes com mais de 35 anos de idade (Poli, et al., 2009; Brasil, 2013).

A pílula combinada é ingerida como um comprimido diário. Existem muitos tipos de pílulas combinadas com diferentes doses e hormônios. Já o anel vaginal tem hormônios similares à pílula combinada. Esse anel é colocado na vagina e permanece ali por três semanas liberando lentamente hormônios que se movem da vagina para a corrente sanguínea. Após este período de três semanas, o anel deve ser retirado e um novo anel é colocado em uma semana depois (Poli, et al., 2009; Brasil, 2013).

A minipílula contém uma forma sintética de apenas um hormônio, a progesterona, e pode não ser tão eficaz quanto à pílula combinada, particularmente em mulheres mais jovens. Necessita ser ingerida todos os dias no mesmo horário e geralmente são mais recomendadas para mulheres que têm efeitos colaterais quando tomam estrogênio ou não podem tomar estrogênio por razões de saúde. A minipílula pode ser usada durante a amamentação (Poli, et al., 2009; Brasil, 2013). Por não conterem estrogênio as minipílulas são indicadas, preferencialmente, em situações em que há contraindicação absoluta ou relativa para o uso de estrogênios, presença de efeitos adversos com o uso do estrogênio ou durante a amamentação. A eficácia contraceptiva é maior durante o período da lactação. Quando as pílulas são tomadas de forma correta, ocorrem menos de uma gravidez para cada 100 mulheres que as usam durante o primeiro ano (nove para cada 1.000 mulheres). A taxa de falha com o uso típico é de 3\% a 5\% (Finotti, 2015).

Sobre os métodos de barreira da contracepção, as opções incluem: preservativo masculino; preservativo feminino e o diafragma. Os preservativos masculinos e femininos também diminuem o risco de infecções sexualmente transmissíveis. Esses preservativos podem ser muito eficazes se usados da maneira certa todas as vezes que acontecer a relação sexual (Poli, et al., 2009; Brasil, 2013).

O preservativo masculino é uma cobertura de látex que é colocada sobre o pênis ereto, impedindo o esperma de entrar na vagina. Esse método é $98 \%$ eficaz se usado da maneira correta. Os preservativos masculinos não são tão caros quantos outros métodos e estão disponíveis em farmácias sem prescrição médica, bem como em supermercados. São também entregues de forma gratuita nas Unidades Básicas de Saúde (Poli, et al., 2009; Brasil, 2013).

O preservativo feminino é uma bolsa solta de látex com um anel flexível em cada extremidade que fica na vagina, impedindo o esperma de entrar no útero e pode ser colocado várias horas antes da relação sexual, por ser mais resistente que o preservativo masculino. O uso deste método pode, quando usado da maneira certa, conferir 95\% de eficácia (Finotti, 2015).

O diafragma é uma cúpula de silicone macia e rasa que se encaixa na vagina. Ele cobre a abertura para o útero, impedindo o esperma de passar. O diafragma pode ficar nessa região por pelo menos seis horas após a relação sexual. Se usado da maneira correta, este método possui uma eficácia de 86\% (Poli, et al., 2009; Brasil, 2013; Finotti, 2015).

Todos os métodos permanentes de contracepção envolvem procedimentos cirúrgicos e são considerados métodos muito eficazes de contracepção. A esterilização feminina, também conhecida como ligadura tubária, envolve a realização de uma 
cirurgia (corte) em cada trompa de falópio (Melo, et al., 2020). A esterilização masculina, ou vasectomia, é uma pequena cirurgia feita com anestesia local em cima do escroto, na qual é cortado o canal que leva os espermatozoides dos testículos até as outras glândulas que produzem o sêmen (Santos, et al., 2020).

A eficácia dos métodos permanentes de contracepção é $99 \%$ e tem como vantagem não possuírem ação hormonal e não geram interações medicamentosas, no entanto, tem como desvantagens o fato de que a reversão dessas cirurgias nem sempre é eficaz, além disso, não protegem contra infecções sexualmente transmissíveis, entre outros riscos de complicações cirúrgicas (Lopes, et al., 2020).

Os métodos naturais ou métodos de conscientização da fertilidade da contracepção incluem o método sintotérmico, a observação do muco cervical e os métodos de temperatura basal. Eles são baseados no monitorando dos sinais corporais diariamente, como alterações na temperatura do corpo e no fluido vaginal. Isto é para determinar quando a mulher está potencialmente fértil durante o ciclo menstrual (Klöppel \& Rohden, 2021).

A prática do uso dos métodos naturais de contracepção requer abstenção ou não ter relações sexuais nos dias indicados, por isso, estes não são recomendados em algumas circunstâncias, como por exemplo: febre, infecção vaginal ou síndrome do ovário policístico. A eficácia do planejamento familiar natural varia, dependendo de qual método ou combinação de métodos usados (Sousa \& Cipriano, 2021).

A contracepção de emergência mais utilizada é a pílula anticoncepcional de emergência, ou comumente chamada de "pílula do dia seguinte". A pílula anticoncepcional de emergência deve ser tomada o mais rápido possível após o sexo desprotegido para garantir máxima eficácia. Atualmente, no Brasil, existem dois tipos de pílulas para a contracepção de emergência: pílulas combinadas que contêm estrogênio e progesterona (método Yuzpe) e pílulas contendo somente progesterona - levonorgestrel, que é a forma mais difundida e utilizada de contracepção de emergência (Sousa \& Cipriano, 2021).

\subsection{Atenção farmacêutica na orientação do uso dos métodos contraceptivos}

O termo atenção farmacêutica surgiu, como uma nova filosofia de prática profissional, provocando um profundo repensar da classe farmacêutica em meados da década de 80 nos Estados Unidos, com a expressão pharmaceutical care. A atenção farmacêutica em outras palavras é a provisão responsável da farmacoterapia, de maneira a alcançar resultados que melhorem a qualidade de vida do paciente (Ranieri \& Silva, 2011).

Em todas as atuações práticas do farmacêutico existem inúmeras oportunidades de auxílio aos pacientes que usam ou procuram usar métodos de contracepção. Nas farmácias das Unidades Básicas de Saúde, os farmacêuticos têm o importante papel de fornecer ao paciente as informações necessárias sobre como usar com segurança e efetivamente os métodos contraceptivos, principalmente durante o atendimento prescritivo (Pereira \& Freitas, 2008).

O envolvimento do farmacêutico com o paciente pode começar durante as visitas à farmácia para obter métodos contraceptivos, como preservativos ou contracepção de emergência ou conversas sobre métodos contraceptivos, à medida que o paciente explora suas possíveis opções. Os farmacêuticos, então, fornecem aconselhamento medicamentoso e educação em saúde à medida que o paciente inicia sua avaliação contraceptiva e contínua adesão e tolerabilidade durante as entregas subsequentes (Soares, et al., 2016).

Os farmacêuticos que trabalham em ambientes hospitalares também estão preparados para auxiliar o paciente e a equipe quanto à seleção de um agente contraceptivo que leve em conta as terapias medicamentosas concomitantes do paciente, potenciais riscos e benefícios da terapia hormonal dadas as condições de comorbidades (Soares, et al., 2016).

Nesse contexto, a atenção farmacêutica quanto a orientações de métodos de contracepção deve começar averiguando o histórico de saúde do paciente. Nessa avaliação o profissional poderá identificar condições ou características relacionadas à segurança do contraceptivo hormonal. É importante que os farmacêuticos façam um questionário de avaliação com critérios de 
triagem para fins de segurança, como por exemplo, confirmar que a paciente não está gravida ou tenha patologias que possam ser agravadas (Melo, 2020).

Faz-se necessário a medição da pressão arterial. A pressão arterial descontrolada ou alta pode representar um risco aumentado ou ser uma contraindicação à seleção do método contraceptivo. O peso não precisa ser verificado, no entanto, em pessoas com o peso elevado, o farmacêutico precisa acompanhar a evolução da alteração do peso (Ranieri \& Silva, 2011).

Ainda em relação à importância da atenção farmacêutica no que diz respeito aos métodos contraceptivos, no Brasil estima-se que em média 30\% das mulheres costuma fazer uso da pílula do dia seguinte, fazendo deste ato um hábito perigoso para a saúde, por isso, a Atenção Farmacêutica contribui de forma significativa no controle da aquisição do contraceptivo, tendo em vista que a utilização deste método tem aumentado o percentual nos últimos anos (Ranieri \& Silva, 2011).

Haja vista, que o farmacêutico é o profissional de saúde que se encontra com maior proximidade ao paciente, além disso, nas farmácias de todo o país são extremamente requisitado nas orientações, pois, dão segurança ao consumidor no momento da compra de alguma medicação, proporcionam confianças ao orientar quanto à utilização correta da substância farmacológica, modo de administração, prescrição, reações adversas, dentre outros. O que garante uma melhor qualidade na saúde do paciente levando a prevenção de problemas maiores, devido ao uso adequado da substância (Matsuoka \& Giotto, 2019).

\section{Conclusão}

Existe uma ampla variedade de medicamentos e métodos empregados na contracepção, e isso pode provocar nos pacientes muitas dúvidas em relação às melhores escolhas ao uso. Por isso o relacionamento entre farmacêutico e paciente tornase imprescindível para uma farmacoterapia bem sucedida.

É fundamental que os farmacêuticos passem informação clara sobre a melhor forma de realizar o tratamento com métodos contraceptivos, além disso, esclareça sobre as formas corretas do uso de medicamentos mostrando também as possíveis contraindicações e interações medicamentosas, contribuindo de forma efetiva, para minimizar os riscos da medicação e reações adversas. A atenção farmacêutica promoverá, portanto, um atendimento personalizado, humanizado e cientificamente correto sobre o uso dos métodos contraceptivos.

Convém destacar que na atualidade, as farmácias e drogarias estão se tornando fundamentais para a ampliação de uma rede de apoio, tendo em vista o vínculo alcançado para com a comunidade que projeta no profissional farmacêutico o anseio para adquirir um diagnóstico favorável à prevenção e recuperação da sua saúde.

Nesse sentido, para que a utilização segura dos métodos contraceptivos seja feitos de forma responsável, os farmacêuticos devem estabelecer vínculos e canais de informação e aconselhamento com a população atendida. Nesse contexto, o profissional farmacêutico passa a ser fundamental, tendo em vista que eles possuem o conhecimento técnico do assunto como prazo e tempo correto para administração do medicamento.

Devido à importância do farmacêutico na comunidade, esse estudo sugere que sejam realizadas outras pesquisas sobre a realizada da atenção farmacêutica em Palmas, Tocantins, para que possa servir de embasamento aos novos profissionais que saem muitas vezes das Instituições de Ensino Superior com uma visão apenas teórica da realidade, no entanto, a prática precisa também ser considerada, para que haja uma maior visibilidade e consequentemente uma valorização profissional para melhorar os processos de trabalho na atenção farmacêutica.

\section{Referências}

Almeida, A. P. F. D., \& Assis, M. M. D. (2017) Efeitos colaterais e alterações fisiológicas relacionadas ao uso contínuo de anticoncepcionais hormonais orais. Rev. Eletrônica Atualiza Saúde, 5(5), 85-93.

Barros, A. J. S., \& Lehfeld, N. A. S. (2000) Fundamentos de metodologia científica: um guia para a iniciação científica. Makron Editora. 
Braga, G. C., \& Vieira, C. S. (2015) Anticoncepcionais reversíveis de longa duração: Implante Liberador de Etonogestrel (Implanon®). Femina, 43 (1), 7-14.

Brandão, E. R. (2019) Métodos contraceptivos reversíveis de longa duração no Sistema Único de Saúde: o debate sobre a (in) disciplina da mulher. Ciênc. saúde colet, 24(3), 875-879.

Brandt, G. P., Oliveira, A. P. R., \& Burci, L. M. (2018) Anticoncepcionais hormonais na atualidade: um novo paradigma para o planejamento familiar. Revista Gestão e Saúde, 18(1), 54-62.

Brasil. (1996) Presidência da República. Lei 9.263, de 12 de janeiro de 1996. Regula o § $7^{\circ}$ do art. 226 da Constituição Federal, que trata do planejamento familiar, estabelece penalidades e dá outras providências. Diário Oficial da República Federal do Brasil, Brasília, DF.

Brasil. (2013) Ministério da Saúde. Saúde sexual e saúde reprodutiva. Cadernos de Atenção Básica n. 26.

Chin, K. L. (2011) Sexual/reproductive health and the pharmacist: what is known and what is needed? Journal of Pharmaceutical Health Services Research, 2(2), 65-69.

Colquitt, C. W., \& Martin, T. S. (2017) Contraceptive Methods. J Pharm Pract, 30(1), 130-135.

Dantas, A. L. B., Carvalho J. G., \& Silva M. D. M. H. (2014) Planejamento familiar: percepção de mulheres que desejam ter filhos. Revista Interdisciplinar, 6(4), 51-59.

Farias, M. R., et al. (2016) Utilização e acesso a contraceptivos orais e injetáveis no Brasil. Revista de Saúde Pública, 50, (2), 1-14.

Finotti, M. (2015) Manual de anticoncepção. São Paulo: Federação Brasileira das Associações de Ginecologia e Obstetrícia (FEBRASGO). https://portaldeboaspraticas.iff.fiocruz.br/wp-content/uploads/2018/07/manual-anticoncepcao-febrasgo-2015-pdf.pdf

Jain, R., \& Muralidhar, S. (2011) Contraceptive methods: needs, options and utilization. The Journal of Obstetrics and Gynecology of India, 61(6), 626-634.

Junges, A. P. P., et al. (2021) Métodos contraceptivos reversíveis de longa ação. Lubianca, J. N., \& Capp, E. (org.). Promoção e proteção da saúde da mulher, ATM 2023/2. Porto Alegre: Universidade Federal do Rio Grande do Sul. Faculdade de Medicina, p. 13-26.

Klöppel, B., \& Rohden, F. (2021) Práticas de percepção da fertilidade entre mulheres jovens. Rev. Estud. Fem, 29(1), e61724.

Leal, A.V., \& Rodrigues, C. R. (2019) Atenção farmacêutica no uso de contraceptivos de emergência: uma breve revisão. Brazilian Journal of Surgery and Clinical Research - BJSCR, 27(2), 159-163.

Lopes, M. M., et al. (2020) Métodos contraceptivos eficácia, vantagens e desvantagens. 2, Canal6. https://www.canal6.com.br/l ivros_loja/EbookMetodoscontraceptivosVolume2.pdf

Matsuoka, J. S., \& Giotto, A. C. (2019). Contraceptivo de emergência, sua funcionalidade e a atenção farmacêutica na garantia de sua eficácia. Revista de Iniciação Científica e Extensão, 2(3), 154-162.

Melo, C. R. M., et al. (2020) Uso de métodos contraceptivos e intencionalidade de engravidar entre mulheres usuárias do Sistema Único de Saúde. Rev. LatinoAm. Enfermagem, 28(e3328), 1-11.

Melo, T. A. R. (2020) Protocolo para prescrição farmacêutica de contraceptivos hormonais em âmbito institucional. (Trabalho de conclusão de curso). João Pessoa: Universidade Federal da Paraíba.

Novosartyan, M. G. (2021) Risks of cerebrovascular disorders associated with combined hormonal contraceptives. Obstetrics, Gynecology and Reproduction, 15(2), 143-155.

Pereira, L. R. L., \& Freitas, O. 2008 A evolução da Atenção Farmacêutica e a perspectiva para o Brasil. Revista Brasileira de Ciências Farmacêuticas, 44(4), 601-612.

Poli, M. E. H., et al. (2009) Manual de anticoncepção da FEBRASGO. Femina, 37(9), 459-92.

Ranieri, C. M., \& Silva, R. F. (2011) Atenção farmacêutica no uso de métodos contraceptivos. (Monografia). Londrina: Centro Universitário Filadélfia de Londrina UNIFIL. https://web.unifil.br/pergamum/vinculos/000003/000003F7.pdf

Santos, D. R., et al. (2020) Crescimento da vasectomia no Sistema Único de Saúde entre 2009 a 2018: um estudo retrospectivo. Revista Eletrônica Acervo Saúde, 12(3), e-2822-e2822.

Soares, L., et al. O devir da profissão farmacêutica e a clínica farmacêutica. Atuação clínica do farmacêutico. Florianópolis: Editora da UFSC; 2016.

Sousa, L. G., \& Cipriano, V. T. F. (2019) Contraceptivo oral de emergência: indicações, uso e reações adversas. Revista Eletrônica Acervo Saúde, 22, e665e665.

Vieira, E. M. (2010) A questão do aborto no Brasil. Revista Brasileira de Ginecologia e Obstetrícia, 32(3), 103-104.

Who. (2019) World Health Organization. Reproductive health. Family planning: a global handbook for providers: evidence-based guidance developed through worldwide collaboration. Johns Hopkins Ccp-Info. 